\title{
Les politiques publiques de développement des milieux ruraux : la Politique nationale de la ruralité du Québec revisitée
}

\author{
Bruno Jean ${ }^{\mathrm{a}}$
}

RÉSUMÉ. Le processus d'élaboration des politiques publiques rurales et l'évolution qu’ont connue ces politiques publiques sont décrits dans le présent article. Leur émergence, relativement récente, a été rendue possible avec la fin de la croyance selon laquelle les politiques sectorielles, notamment agricoles, pouvaient solutionner les problèmes ruraux. Ensuite, nous présenterons plus en profondeur l'expérience québécoise de soutien au développement des territoires ruraux avec la Politique nationale de la ruralité (PNR) du Québec; nous mettrons l'accent sur les aspects novateurs d'une telle politique pour tirer quelques enseignements de sa mise en œuvre de 2001 à 2014 . À plus d'un titre et comme l'a reconnu l'OCDE, cette politique originale a montré la pertinence de s'intéresser aux facteurs intangibles, par exemple la mobilisation collective, l'engagement citoyen et la gouvernance locale, qui sont souvent des déterminants décisifs de l'évolution socioéconomique des milieux ruraux.

ABSTRACT. This article describes the process of developing rural public policies and their evolution. Their relatively recent emergence occurred with the ending of the belief that sectoral policies, particularly agricultural policies, could solve rural problems. The Quebec experience to support the development of rural territories with the National Policy on Rurality (NPR) of Quebec is subsequently discussed in depth. The focus is on the innovative aspects of such a policy, to draw lessons from its implementation from 2001 to 2014. As recognized by the OECD, this original policy has shown on several accounts the relevance of focusing on intangible factors such as collective mobilization, civic engagement and local governance, that are often decisive determinants of socio-economic development in rural areas.

\section{Introduction}

La formulation et la mise en œuvre des politiques publiques rurales ne remontent pas loin dans l'histoire du Québec. Il fallait que le processus de construction sociale de la ruralité comme problème pour lequel une politique publique se présente comme une solution ait produit une prise de conscience partagée entre les citoyens ruraux et urbains et les instances politiques.

Dans un premier temps, nous analyserons ici ce processus d'élaboration des politiques publiques rurales et l'évolution qu'ont connue ces politiques publiques. Ensuite, nous présenterons plus en profondeur l'expérience québécoise de soutien au développement des territoires ruraux avec la Politique nationale de la ruralité (PNR) du Québec en mettant l'accès sur les aspects novateurs d'une telle politique pour tirer quelques enseignements de sa mise en œuvre de 2001 à 2014.

\section{Sur l'émergence des politiques publiques rurales}

Lorsque le Québec était une société majoritairement rurale, il n'y avait pas à proprement parler de politiques rurales, mais une politique agricole et forestière qui prétendait s'intéresser aux problèmes ruraux puisque la ruralité de l'époque était largement agricole et forestière. Paradoxalement, c'est au moment où la ruralité est devenue marginale (avec l'urbanisation massive) qu'on voit apparaittre des politiques rurales se désignant comme telles et se présentant comme distinctes des politiques agricoles. Cette intervention des gouvernements répondait à une demande d'assistance des ruraux.

a Professeur émérite, Université du Québec à Rimouski 
Dans le cas québécois, celle-ci s'est fortement manifestée avec la tenue des États généraux du monde rural en 1991, qui allait donner naissance à Solidarité rurale du Québec, un organisme voué à la défense des intérêts des ruraux. L'intervention de l'État a aussi été légitimée par la mise en œuvre de grands principes démocratiques comme la justice sociale, qui ne peut tolérer de trop grands écarts de développement au sein des différents territoires d'un même espace national. Par ailleurs, on a graduellement reconnu que des campagnes vivantes profitent à toute la société par la production de nombreux biens, mais aussi en lui procurant de nombreux services environnementaux et récréotouristiques.

$\mathrm{Au}$ Québec, les premières politiques rurales ont consisté en des politiques d'ouverture au peuplement de plusieurs régions en dehors du Québec central de la vallée du Saint-Laurent. On parlait alors de colonisation et, pendant longtemps, le gouvernement du Québec avait un ministère de la Colonisation, qui a survécu jusque dans les années 1960, où il a été intégré au ministère de l'Agriculture. À la fin des années 1950, le gouvernement fédéral s'intéresse à la ruralité avec son ministère des Forêts et du Développement rural et, surtout, avec la reconnaissance qu'il existe une "pauvreté rurale » dans plusieurs régions souvent éloignées de plusieurs provinces. Il met donc en place, en 1961, la Loi sur l'aménagement rural et le développement agricole (ARDA), qui autorise la signature d'ententes fédérales-provinciales pour soutenir le relèvement économique de ces territoires. On assiste alors à un autre paradoxe: alors que ce territoires sont largement ruraux, c'est du côté des théories et des modèles de « développement urbain et régional » que les plans de développement élaborés et mis en œuvre trouveront leur inspiration (Lapping et Fuller, 1985).

Au Québec comme dans la plupart des pays dits avancés, la question rurale a toujours été imbriquée avec la question régionale. Ce sont les diverses politiques dites de développement régional des gouvernements fédéral et provincial qui devaient assurer le relèvement des régions rurales en difficulté. Au palier fédéral, cette approche a connu ses belles années avec la création, en 1969, d'un ministère de l'Expansion économique régionale (le MEER), démantelé en 1978 et remplacé par des agences dans différentes régions du pays, comme l'Agence de promotion économique du Canada atlantique (APECA) et l'agence Développement économique Canada pour les régions du Québec (DEC).

Par ailleurs, il s'en est suivi une période où l'on voit apparaitre des politiques publiques spécifiques rurales, dont la Politique nationale de la ruralité (PNR) du Québec en 2001 et la mise en place du Secrétariat rural rattaché à Agriculture Canada en 2003. Toutefois, le nouveau gouvernement du Québec, dirigé par le libéral Philippe Couillard, abolit la PNR en 2014. Au même moment, le gouvernement fédéral achève son implication dans le dossier rural par la fermeture du Secrétariat rural, qui a connu de belles années avec son concept de lentilles rurales (rural lens), qui consistait en une sorte de clause rurale pour s'assurer que les interventions des différents ministères et agences ne produisent pas des effets négatifs dans les milieux ruraux.

La Politique nationale de la ruralité est donc arrivée à un moment donné d'un processus de reconnaissance sociale et politique des problèmes ruraux exprimés depuis les années 1950, et face auxquels diverses approches d'intervention publique ont été expérimentées. Sommairement, l'évolution des politiques publiques mises en œuvre pour soutenir le développement rural a suivi à peu près les étapes suivantes :

Les années 1950 : On assiste à une prise de conscience des problèmes ruraux, lesquels sont identifiés comme des problèmes de pauvreté rurale mesurés par le gouvernement fédéral grâce à divers indicateurs socioéconomiques, mais il s'agit d'une approche sectorielle. Puisque le rural est agricole, avec la politique agricole - une politique sectorielle -, on prétendait régler les éventuels problèmes ruraux. On voit aussi apparaittre des interventions libellées comme des programmes de "développement régional » pour les régions rurales en difficulté. Ces interventions misaient sur l'effet structurant des «pôles de croissance » urbains. Malgré des efforts importants pour mettre en place des infrastructures dans ces régions, les effets attendus des pôles de croissance n'ont pas engendré les résultats escomptés.

Les années 1960-1970 : On assiste à la prise de conscience du maintien des disparités régionales de développement et à la nécessité de répondre à la demande de parité des ruraux. Un véritable mouvement social 
rural a émergé avec les luttes de résistance des ruraux de l'Est-du-Québec avec les Opérations-Dignités. La réponse étatique a consisté en l'accélération de la mise en place d'infrastructures de toutes sortes pour le développement économique (autoroutes, aéroports, etc.) et pour le développement humain (écoles, universités, hôpitaux, etc.).

Les années 1980 : On assiste à une prise de conscience selon laquelle les politiques sectorielles (agricoles, forestières, industrielles, etc.) ne peuvent servir de politiques de développement rural et que la ruralité, devenue une réalité complexe, nécessite ses propres politiques publiques. Les politiques publiques prennent alors un virage vers le développement local. Le soutien aux initiatives locales a le mérite de coûter moins cher, mais de produire autant de résultats positifs, tout en mobilisant les populations locales, comme c'est le cas avec le programme LEADER en Europe, le Programme de développement des collectivités (PDC) ayant donné lieu aux sociétés d'aide au développement des collectivités (SADC) au Canada et le Rural Partnership aux États-Unis.

Des éléments de convergence se dégagent de ces trois grandes politiques : l'approche territoriale et ascendante; la concertation et le partenariat avec les instances locales; la décentralisation administrative; l'orientation vers la revitalisation du rural fragilisé; la sensibilité à la formation, à l'innovation et à la transférabilité des expériences novatrices; et, finalement, le développement des capacités des collectivités.

Les années 1990 à nos jours: On assiste à une double tendance, soit l'apparition des premières politiques rurales spécifiques (en Finlande, au Japon et en France), soit un engouement pour le développement fondé sur les lieux (place-based development) dans un contexte où le développement basé sur les personnes (people-based development) est largement pratiqué par les politiques publiques. Au Québec, la première véritable politique rurale privilégiant cette approche de mobilisation locale apparaît en 2001, quelques années après la mise en place d'un réseau de centres locaux de développement (CLD) sur la base des territoires de MRC qui seront mobilisés pour appliquer cette politique. Or, les politiques rurales sont parfois remises en question par des observateurs qui jugent ces politiques trop centrées sur le rural, et pas assez sur les liens ville-campagne. Une telle observation ne s'applique pas à la PNR puisqu'elle s'est appuyée largement sur la nouvelle approche théorique du développement territorial que le Centre de recherche sur le développement territorial (CRDT) a contribué à mettre en forme (Jean, 2008).

Ce nouvel intérêt des gouvernements occidentaux pour la question rurale s'est transporté dans une agence intergouvernementale comme l'Organisation de coopération et de développement économiques (OCDE), dont les membres sont les 27 pays les plus développés de la planète. De nombreuses publications sur les politiques publiques émanent de cette organisation. On lui doit des réflexions fort bien documentées (OCDE, 2004, 2005) sur divers problèmes ruraux, notamment sur la nécessité d'un changement de paradigme pour penser l'action publique dans les territoires ruraux. En gros, il s'agirait pour les gouvernements de changer leurs représentations du rural comme un milieu qui a besoin d'assistance (ou de subsides pour compenser les handicaps) à un milieu qui a des atouts et des actifs de toutes sortes dans lesquels les gouvernements peuvent faire des investissements pour assurer le développement des communautés rurales par la valorisation des ressources naturelles de ces milieux.

Les politiques rurales opèrent souvent selon une logique compensatrice. Selon cette vision, les espaces ruraux souffrent d'un écart de croissance par rapport aux pôles urbains en raison de handicaps principalement dus aux effets de la distance des marchés et des centres de services. Les interventions publiques doivent alors combler ces handicaps de localisation, de ressources humaines ou d'équipement qui limitent l'attractivité de ces territoires.

Toutefois, d'autres politiques rurales optent parfois pour une logique allocatrice. Dans cette perspective, chaque territoire est censé receler ses propres facteurs de développement économique et social : identité, ressources naturelles et humaines, patrimoine paysager et culturel, savoir-faire. Les défaillances du marché, justifiant l'intervention publique, résultent des difficultés de circulation de l'information sur les occasions à saisir ainsi que de la faible mobilité du capital, qui gênerait l'adaptation des systèmes productifs ruraux.

Finalement, plusieurs politiques rurales misent sur une dimension organisationnelle, soit le renforcement des capacités de développement des milieux 
ruraux eux-mêmes, qui sont ainsi appelés à se mobiliser et à sortir de la mentalité d'assistés. Avec le concept de gouvernance locale, on pense que, si de nouveaux mécanismes de prise de décision sur les affaires collectives se basent sur le partenariat et la concertation entre le secteur public, le secteur privé et la société civile, ce nécessaire « renforcement des capacités de développement » des communautés rurales pourra se produire (Jean, Épenda Muteba Wa, 2004). Quand on considère la politique rurale du Québec, son orientation générale va justement dans le sens du renforcement des capacités des acteurs locaux de développement et de la création d'une nouvelle gouvernance, en misant sur la présence d'un capital social et, donc, sur ce qu'on peut appeler des facteurs intangibles ou immatériels de développement.

\section{La Politique nationale de la ruralité du Québec}

La Politique nationale de la ruralité (PNR) est officiellement annoncée le 6 décembre 2001' Comme il est admis chez les politologues, pour qu'une politique publique soit adoptée, il faut trois prérequis : 1) un problème; 2) un contexte favorable; et 3) une solution. À quel problème la PNR présentée comme solution pouvait-elle bien répondre?

La reconnaissance d'un problème rural a été une tâche ardue à laquelle s'est attaquée une coalition d'acteurs de la ruralité réunis au sein de Solidarité rurale du Québec (SRQ). Le problème doit aussi être attesté par la littérature scientifique. Le gouvernement disposait alors, entre autres, d'un livre que nous avions écrit quelques années auparavant (Jean, 1997). Il aura fallu une dizaine d'années pour définir le problème et mettre en place une solution telle que la PNR. Le contexte favorable aura sûrement été l'arrivée au pouvoir du Parti québécois, soucieux de respecter les demandes de sa base électorale et de répondre aux attentes de certains leaders de SRQ sympathisants ou membres du parti.

Dans l'énoncé de cette première politique rurale, on retrouve une description du problème ou plutôt cinq grands défis ou enjeux qu'affrontent les milieux ruraux, soit 1) l'évolution démographique et l'occupation du territoire; 2) la diversification économique et la mise en valeur des ressources; 3) l'amélioration de la qualité de vie; 4) la participation et la prise en main par le milieu; et, finalement, 5) l'adaptation des interventions gouvernementales à la réalité du monde rural.

Les interventions gouvernementales envisagées au titre de solution sont définies comme trois grandes orientations qui se déclinent ainsi : 1) stimuler et soutenir le développement durable et la prospérité des collectivités rurales; 2) assurer la qualité de vie des collectivités rurales et renforcer leur pouvoir d'attraction; et 3) soutenir l'engagement des citoyens et citoyennes au développement de leur communauté et assurer la pérennité du monde rural.

Une nouveauté importante de la PNR était la reconnaissance de la MRC comme palier de gouvernement local responsable de la mise en ouvre de la PNR dans le cadre d'une entente contractuelle dont les termes étaient définis dans un pacte rural. On convenait que la MRC s'engageait à entreprendre une démarche de réflexion, d'organisation et d'action au sein de chaque communauté rurale et, au sein de la MRC, à appuyer les opérations menant à la réalisation de projets, et à identifier et établir les initiatives pour soutenir les actions des communautés du territoire visé. Pour obtenir des fonds au titre de la PNR, les MRC devaient suivre un plan de travail accepté par le gouvernement.

Cette première PNR, prévue de 2001 à 2007, visait, selon nous, le renforcement des capacités de développement des communautés rurales et, donc, des facteurs intangibles de développement local (Jean, 2002). Cette politique rurale reposait donc sur un modèle causal implicite de développement rural misant sur les forces existantes dans les milieux (les différents capitaux) pour assurer la mobilisation des citoyens et accroitre leurs capacités de développement en vue d'un développement durable des collectivités. Le graphique 1 rappelle ce modèle causal anticipé par la PNR. 


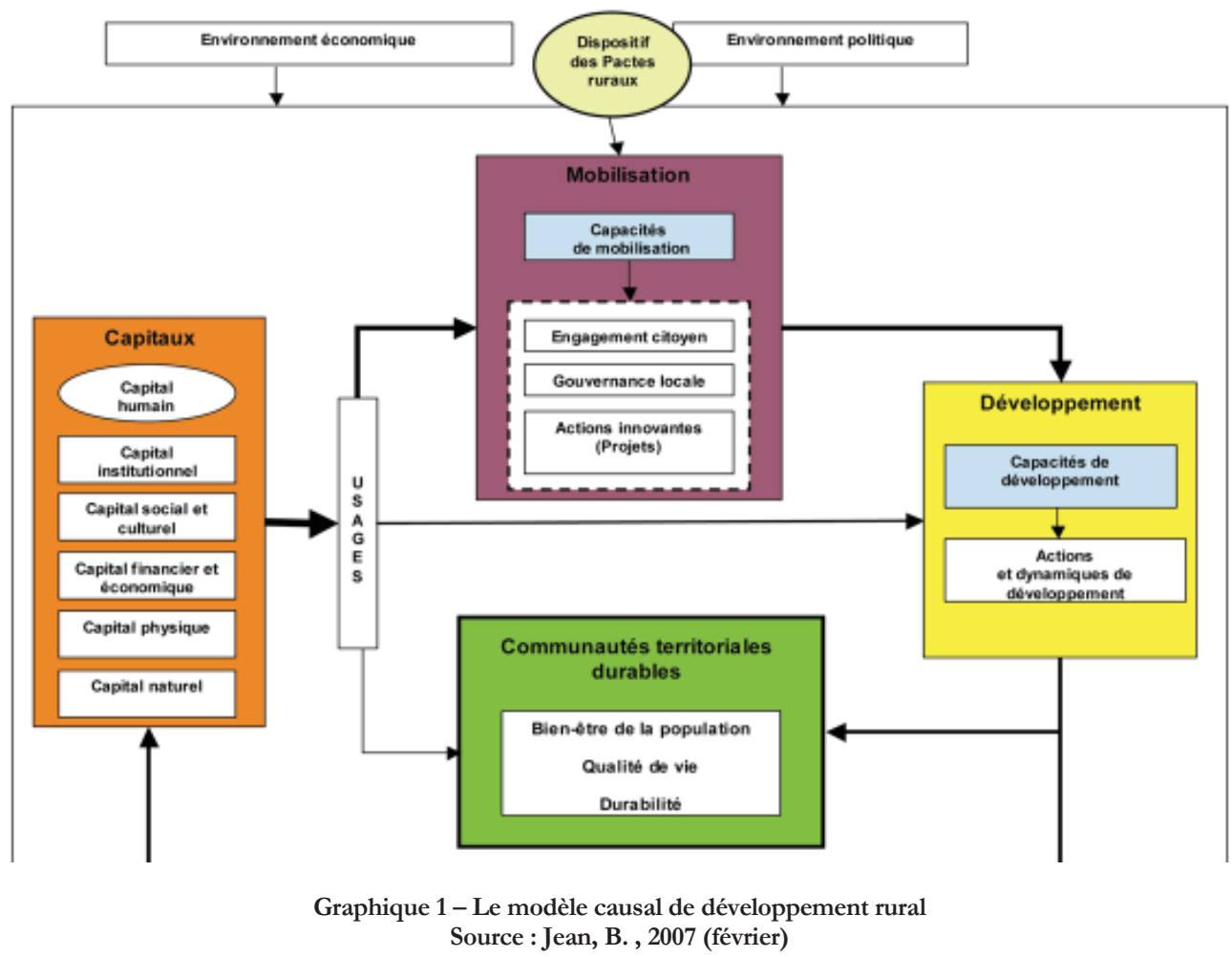

Puisque la première PNR a connu un succès reconnu par les élus locaux tout comme les études évaluatives objectives, le gouvernement libéral de Jean Charest l'a reconduite, et ce, pour une période d'application prolongée de 2007 à 2014. Cette PNR2 identifie d'abord quatre « défis et orientations » propres à cette seconde phase de la Politique rurale du Québec : 1) promouvoir le renouvellement et l'intégration des populations; 2) favoriser la mise en valeur des ressources humaines, culturelles et physiques du territoire; 3) assurer la pérennité des communautés rurales; et 4) maintenir un équilibre entre qualité de vie, cadre de vie, environnement naturel et activités économiques.

La PNR2 innove aussi avec la proposition de soutenir significativement des initiatives expérimentales de développement des milieux ruraux avec des laboratoires ruraux. L'objectif était de soutenir financièrement 25 expériences approfondies de développement rural dans des secteurs à potentiel peu exploré jusqu'à maintenant, avec obligation de transfert de connaissances et transmission du savoir-faire. L'aide financière pouvait atteindre 100000 \$ par année pour cinq ou six ans.

Plus de 30 projets ont été financés dans plusieurs domaines, dont la santé en milieu rural (4 projets); l'agriculture innovante de spécialité et de proximité
(4); la nouvelle foresterie (3); le développement intégré des ressources en territoire rural (3); l'école au cœur de la communauté (2); la culture et le patrimoine au cœur du développement rural (2); les milieux ruraux comme acteurs dans le domaine énergétique (2); l'accueil, l'installation et l'intégration de nouvelles populations (1) ainsi que la communauté entrepreneuriale et l'entrepreneuriat collectif (1).

La PNR2 a aussi innové en mettant en place des groupes de travail, dont le mandat était d'étudier ou d'explorer des pistes d'avenir, puis de les transférer aux milieux ruraux en réunissant des ressources humaines et des connaissances sur chaque thème. Six groupes de travail ont été mis en place et ont produit des études fort intéressantes sur six thèmes concernant la ruralité québécoise : 1) le milieu rural comme producteur d'énergie; 2) la multifonctionnalité des milieux ruraux; 3) la mise en marché des produits de spécialité : les emporiums du Québec; 4) le branchement aux réseaux de télécommunication; 5) la complémentarité rurale-urbaine; et 6) les milieux dévitalisés.

Avec l'annonce de la PNR3 en 2014, le langage évolue et de nouveaux concepts apparaissent, traduisant de nouvelles préoccupations de gouvernance, d'approche intersectorielle, de développement multifonctionnel et de complémentarité rurale-urbaine. Cette 
PNR3 identifie six grands principes directeurs qui vont inspirer l'action publique : 1) s'appuyer sur les avancées des politiques précédentes; 2 ) miser sur une plus grande décentralisation; 3) promouvoir l'approche intersectorielle, la multifonctionnalité du développement et les initiatives de complémentarité et urbaine; 4) maintenir la souplesse d'application et le respect de l'autonomie locale dans les choix de mise en œuvre; 5) encourager la participation citoyenne; et 6) préconiser une approche solidaire et équitable sur le plan territorial.

Alors qu'elle est prévue pour 10 ans, le nouveau gouvernemental libéral abolit cette politique rurale en 2014, soit peu de temps après son lancement. Il s'agit d'un virage inattendu (Jean, 2015). Les coupes concernent plusieurs autres dispositifs de développement régional et local au Québec. Cela signifie la fin de la reconnaissance et du financement de Solidarité rurale du Québec, qui avait réussi à porter dans le système gouvernemental et dans la plus large partie de la société québécoise les attentes et les préoccupations des populations rurales.

\section{Les enseignements de la PNR sur l'action publique dans les territoires ruraux}

La PNR a opéré un véritable changement de paradigme de l'action publique en reconnaissant la nécessité de changer nos représentations de la ruralité comme un milieu qui a besoin d'assistance (une dépense) pour compenser ses handicaps à une vision du rural comme un milieu qui a des atouts et des potentialités pour lesquels un développement est possible, si on y consacre des investissements publics.

Ce changement de paradigme a été reconnu par l'OCDE, qui a salué la pertinence et l'originalité de la PNR (OCDE, 2010). Malgré les retombées positives de la PNR (Jean, Desrosiers et Dionne, 2014), l'opinion publique considère encore les investissements publics en faveur du développement rural comme une dépense. Une prétendue crise dans les finances publiques peut alors légitimer, comme l'a fait le gouvernement libéral de Philippe Couillard en 2014, une coupe radicale dans ce secteur.

L'action publique rurale avec l'arrivée d'une politique publique qui cible des facteurs intangibles de développement local (p. ex. : renforcement des capacités de développement des communautés, engagement citoyen, mobilisation, empowerment, etc.) représente un changement de paradigme qui se distingue fondamentalement de l'action publique, qui investit exclusivement dans les infrastructures.

La PNR que s'était donnée le gouvernement du Québec était novatrice à plusieurs points de vue. Avec cette politique, un gouvernement central était capable de reconnaitre la capacité d'action des gouvernements locaux en leur déléguant la mise en œuvre des principaux dispositifs de cette politique. Donc, au lieu d'une action publique traditionnellement descendante (top-down), on pratiquait une approche ascendante (bottom-up). Cette politique était aussi originale par les dispositifs ou les outils mis en place. Par exemple : les laboratoires ruraux, qui ne sont pas sans faire penser aux living labs populaires de nos jours, la mise en place d'un pacte rural avec chaque MRC, le soutien à un réseau d'agents de développement rural et la modulation du financement alloué en fonction du niveau de défavorisation des collectivités rurales de la MRC.

Avec les États généraux du monde rural de 1991 et la montée en puissance de Solidarité rurale du Québec, la question rurale est devenue un objet de sollicitude des pouvoirs publics. Or, au moment où tout le monde reconnaissait les effets bénéfiques des investissements publics en faveur du développement rural, un nouveau gouvernement a mis fin à cette politique.

Un problème reste entier : qui peut aujourd'hui prétendre porter la voix d'une ruralité aux multiples groupes sociaux et aux intérêts souvent antagoniques? La diversité n'est pas seulement un phénomène urbain. La ruralité aussi a droit à sa diversité. Actuellement, un organisme comme la Fédération des municipalités du Québec, forte de sa légitimité car elle représente pratiquement tous les élus locaux des municipalités rurales du Québec, tente de porter cette voix rurale par la mise en place d'une table de concertation rurale, qui tentera de renouer un dialogue avec le gouvernement du Québec.

Malheureusement, la question rurale ne semble plus à l'agenda des préoccupations des gouvernements, qui se sont plutôt désengagés. Seul un fort revirement de l'opinion publique, qui pourrait émerger avec la sensibilité environnementale actuelle, pourrait réactiver une demande sociale pour des politiques publiques rurales adaptées aux défis d'un développement durable des milieux ruraux. 


\section{NOTES}

1 Une bonne partie des propos exposés ici relèvent d'une observation participante de la mise en œuvre de cette politique, et non d'une méta-analyse de la littérature scientifique, d'ailleurs fort peu abondante, sur le sujet.

\section{RÉFÉRENCES}

Agricultural and Rural Restructuring Group. (1994). Toward a whole rural policy for Canada, ARRG Working Paper 7. Brandon, MB : Rural Development Institute.

Bollman, R. D. (1992). Régions rurales et petites villes du Canada. Toronto, ON : Thompson Educational Publishing.

Douglas, D. (2010). Rural planning and development in Canada. Toronto, ON : Nelson Education.

Jean, B. (1997). Territoires d'avenir : pour une sociologie de la ruralité. Québec, QC : Presses de l’Université du Québec.

Jean, B. (2002). La reconnaissance politique de la ruralité québécoise. Dans R. Côté et M. Venne (dir.). L'annuaire du Québec 2003 (p. 231-241). Montréal, QC : Fides.

Jean, B. (2007, février). Le modèle causal de développement rural au Québec. Dans P. Leblanc, (dir.), La mise en application et l'évaluation du dispositif du Pacte Rural (p. 51-70). Rapport de recherche 1, Rimouski, QC : CRDT/UQTR.

Jean, B. (2008). Le développement territorial : une discipline scientifique émergente. Dans G. Massicotte (dir.), Sciences des territoires : perspectives québécoises (p. 283-313). Québec, QC : Presses de l’Université du Québec.

Jean, B. (2015). Le désengagement de l'État envers la ruralité : un virage inattendu. Dans A. Poitras (dir.), L'état du Québec 2016 (p. 267-273). Montréal, QC : Institut du Nouveau Monde/Del Busso.

Jean, B., Desrosiers, L. et Dionne, S. (2014). Comprendre le Québec rural (2e éd. revue et augmentée). Rimouski, QC : Université du Québec à Rimouski, Chaire de recherche du Canada en développement rural, Groupe de recherche interdisciplinaire sur le développement régional de l'Est du Québec, Centre de recherche sur le développement territorial. Repéré à http://semaphore.uqar.ca/856/1/UQAR_LivreQuebecRural_v6.pdf

Jean, B. et Épenda Muteba Wa, A. (2004). Le capital social et le développement des « capacités de développement » des communautés rurales : les enseignements d'une étude canadienne. Revue d'Économie régionale et urbaine, 3, 673-694

Lafontaine, D. et Boisjoly, J. (2007). La mise en application et l'évaluation du dispositif du Pacte rural, rapport de recherche 2. Rimouski, QC : UQAR, Centre de recherche sur le développement territorial.

Lapping, M. B. et Fuller, A. M. (1985). Rural development policy in Canada: An interpretation. Community Development Journal, 20(2), 114-119.

Organisation de coopération et de développement économiques (OCDE). (1990). Les partenaires pour le développement rural. Paris : OCDE.

Organisation de coopération et de développement économiques (OCDE). (1993). Quel avenir pour nos campagnes? Une politique de développement rural. Paris : OCDE.

Organisation de coopération et de développement économiques (OCDE). (2004). Place-based policies for rural development: The micro-regions strategy, Mexico (case study). Paris : OCDE. Repéré à www.oecd.org/regional/regional-policy/34857346.pdf

Organisation de coopération et de développement économiques (OCDE). (2005). New approaches to rural policy: Lessons from around the world. Paris: OCDE. https://doi.org/10.1787/9789264010130-en

Organisation de coopération et de développement économiques (OCDE). (2010). Examens de l'OCDE des politiques rurales : Québec, Canada. Paris : OCDE. 